\title{
Naturally convective Casson fluid flow in presence of nano particle through porous plate with thermal radiation and MHD effects
}

\author{
Khadiza Begum ${ }^{1}$, Ahammad Hossain ${ }^{2}$, Farhana Mamtaz ${ }^{3}$,Nusrat Sharmin ${ }^{4}$,Abu \\ Hanif Sarkar ${ }^{5}$ \\ ${ }^{1}$ Department of Mathematics, Prime University, Dhaka, \\ ${ }^{2}$ Department of Mathematics, Sonargaon University, Dhaka, \\ ${ }^{3}$ Department of Mathematics, CSUN, USA, \\ ${ }^{4}$ Department of Mechanical Engineering , Sonargaon University, Dhaka, \\ ${ }^{5}$ Department of Mathematics, Jagannath University, Dhaka,
}

\begin{abstract}
Naturally convective and thermally radiative MHD Casson fluid with nano particle flow through a vertical porous plate has been studied. A flow model is established by employing the well-known boundary layer approximations. In order to obtain non-dimensional system of equations, a similarity transformation is applied on the flow model. The coupled non-linear partial differential equations are solved by explicit finite difference method and the numerical results have been calculated by Compaq Visual 6.6a. The effects of various parameters entering into the problem on velocity, temperature and concentration are shown graphically.
\end{abstract}

Keywords: Casson fluid, Nano particle, Porous Plate, MHD, Thermal Radiation, Skin friction, Nusselt number, Local Sherwood number, Heat and Mass Transfer.

\section{Introduction}

The fluid which is a shear thinning liquid having an infinite viscosity at zero rate of shear, no flow happens yield stress below is called Casson fluid. Magnetohydrodynamics Casson fluid with nano particles through stretching sheet plays a vital rule in the view of its wide applications in many engineering, heating elements and nano technology, chemical engineering, materials engineering, metallurgy, extrusion of polymer, cooling of metallic plate, food, drilling operations and bio- engineering. The quality of the resulting product depends on the heat transfer rate, and so, the knowledge of the flow and heat transfer properties of the ambient fluids are very much essential. Ibukun et al. [1] and Reddy et al. [2] investigated the combined Dufour and Soret effects on the heat and mass transfer in a Casson nano-fluid flow over an unsteady stretching sheet with thermal radiation and heat generation. The study of the fluid flow, heat and mass transfer in a Casson nano-fluid, the Casson fluid being the base fluid has been investigate. The traditional Casson nano-fluid model is revised to include the effect of thermophoresis and Brownian motion. The Casson fluid flow technology is used for polymer processing, manufacturing of glass sheets, paper production, in textile industries and many others engineering. Nadeem et al. [3] and Reddy [4] initiated a study on the boundary layer flow of a viscous fluid towards a linear stretching sheet. The boundary layer MHD flow of Casson fluid induced by an exponentially shrinking sheet by Adomian Decomposition Method and shooting method with fourth order Runge-Kutta scheme have been employed to obtain the analytical and numerical solution. The unsteady two-dimensional flow of a non-Newtonian fluid over a stretching surface having a prescribed surface temperature is investigated. The Casson fluid model is used to characterize the non-Newtonian fluid behavior. Mukhopadhyay et al. [5] investigated the Casson fluid flow over an unsteady stretching surface. Srinivasa et al. [6] studied heat generation effect of electrically conducting fluid flow towards an isothermal truncated cone. Hayat et al. [7] analytically investigated the effect of existence of heat generation/absorption on MHD three-dimensional Oldroyd-B nanofluid flow. Couette flow in a rectangular channel of an electrically conducting viscous fluid under the action of a transversely applied magnetic field has immediate applications in many devices such as magnetohydrodynamic (MHD) power generators, MHD pumps, accelerators, aerodynamics heating, electrostatic precipitation, polymer technology, and petroleum industry, purification of crude oil and fluid droplets sprays. Ahmed et al. [8] and Mosayebidorcheh et al. [9] studied the time dependent pressure gradient effect on unsteady MHD Couette flow and heat transfer of a Casson Fluid. The development of the slip effects on the boundary layer flow and heat transfer over a stretching surface in the presence of nano particle fractions. Effect of partial slip boundary condition on the flow and heat transfer of nano fluids past stretching sheet prescribed constant wall temperature has been investigate by Noghrehabadi et al. [10]. Gaffar et al. [11] investigated the nonlinear steady laminar boundary layer flow and heat transfer of an incompressible Tangent 
Hyperbolic non-Newtonian fluid from a sphere. The transformed conservation equations are solved numerically using implicit finite-difference Keller Box technique.

The studies concern with the numerical investigation of Casson fluid in presence of nano particles with thermal radiation, MHD, porous plate. This work presents unsteady MHD free convective flow past a vertical porous plate. The governing coupled non-linear partial differential equations are first transformed into a dimensionless momentum, angular momentum, energy and concentration equations and then the resultant nonlinear set of equations has been solved numerically employing explicit finite difference technique. From the physical point of view, the numerical results for various parametric values have been presented graphically.

\section{Mathematical formulation}

Naturally convective Casson fluid flow along a semi-infinite vertical porous plate $y=0$ is considered in the presence of a uniform thermal radiation, nano particles and magnetic field. The flow is considered to be in the $x$-direction which is taken along the plate in the upward $x$-direction and $y$-axis is normal to it. When, the plate velocity $U(t)$ is given as $u=U_{0}$. In initial step, it is considered that the plate as well as the fluid particle is at rest at the same temperature $T\left(=T_{\infty}\right)$ and the same concentration level $C\left(=C_{\infty}\right)$ at all points.

Continuity Equation:

$\frac{\partial u}{\partial x}+\frac{\partial u}{\partial y}=0$

Momentum Equation:

$\frac{\partial u}{\partial t}+u \frac{\partial u}{\partial x}+v \frac{\partial u}{\partial y}=g \beta\left(T-T_{\infty}\right)+g \beta^{*}\left(C-C_{\infty}\right)+v\left(1+\frac{1}{\alpha}\right) \frac{\partial^{2} u}{\partial y^{2}}-\frac{\sigma^{\prime} B_{0}^{2} u}{\rho}-\frac{v}{K^{\prime}} u$

Energy Equation:

$\frac{\partial T}{\partial t}+u \frac{\partial T}{\partial x}+v \frac{\partial T}{\partial y}=\frac{\kappa}{\rho c_{p}} \frac{\partial^{2} T}{\partial y^{2}}+\frac{v}{c_{p}}\left(1+\frac{1}{\alpha}\right)\left(\frac{\partial u}{\partial y}\right)^{2}-\frac{1}{\rho c_{p}} \frac{\partial q_{r}}{\partial y}$

$+\tau\left\{D_{B}\left(\frac{\partial T}{\partial y} \frac{\partial C}{\partial y}\right)+\frac{D_{T}}{T_{\infty}}\left(\frac{\partial T}{\partial y}\right)^{2}\right\}$

Concentration Equation:

$\frac{\partial C}{\partial t}+u \frac{\partial C}{\partial x}+v \frac{\partial C}{\partial y}=D_{B}\left(\frac{\partial^{2} C}{\partial y^{2}}\right)+\frac{D_{T}}{T_{\infty}} \frac{\partial^{2} T}{\partial y^{2}}$

With boundary condition,

$u=U_{0}=b x, v=0, T=T_{w}, C=C_{w}$ at $y=0$

$u=0, T \rightarrow T_{\infty}, C \rightarrow C_{\infty}$ at $y \rightarrow \infty$

Where, $u$ and $v$ are the velocity component, $B_{0}$ is the magnetic field component, $\beta$ is thermal expansion coefficient, $\beta^{*}$ is concentration expansion coefficient, $T_{w}$ denotes the wall temperature, $C_{w}$ is the species concentration at the wall, $b$ is the stretching constant, $v$ is the kinematic viscosity, $\rho$ is density, $\kappa$ is thermal conductivity, $c_{p}$ is specific heat at constant pressure, $q_{r}$ unidirectional radiative heat flux, $D_{B}$ is Brownian diffusion coefficient, $D_{T}$ thermophoresis diffusion coefficient, $\tau=(\rho c)_{p} /(\rho c)_{f}$ is the ratio of the heat capacity of the nano particle material and the heat capacity of the fluid and $\alpha=k_{c} \sqrt{2 \pi_{c}} / \tau_{0}$ is the non-Newtonian Casson parameter. The radiative heat flux term by using the Rosseland approximation is given by $q_{r}=-\frac{4 \sigma_{s}}{3 k_{e}} \frac{\partial T^{4}}{\partial y}$.

Where, $\sigma_{s}$ is the Stefan-Boltzmann constant and $k_{e}$ is the mean absorption coefficient, respectively. If temperature differences within the flow are sufficiently small, then the $q_{r}$ can be linearized by expanding $T^{4}$ into the Taylor series about $T_{\infty}$, which after neglecting higher order terms takes the form by $T^{4} \cong 4 T_{\infty}^{3} T-3 T_{\infty}^{4}$. Then the equation (3) becomes 


$$
\begin{aligned}
& \frac{\partial T}{\partial t}+u \frac{\partial T}{\partial x}+v \frac{\partial T}{\partial y}=\frac{\kappa \partial^{2} T}{\rho c_{p} \partial y^{2}}+\frac{v}{c_{p}}\left(1+\frac{1}{\alpha}\right)\left(\frac{\partial u}{\partial y}\right)^{2}+\frac{16 \sigma_{s} T_{\infty}^{3}}{3 k_{e} \rho c_{p}} \frac{\partial^{2} T}{\partial y^{2}} \\
& +\tau\left\{D_{B}\left(\frac{\partial T}{\partial y} \frac{\partial C}{\partial y}\right)+\frac{D_{T}}{T_{\infty}}\left(\frac{\partial T}{\partial y}\right)^{2}\right\}
\end{aligned}
$$

From the governing equations (1) - (5) under the initial conditions and the boundary conditions will be based on the finite difference method it is required to make the equations dimensionless. For the purpose introducing the following dimensionless quantities:

$$
X=\frac{x U_{0}}{v}, Y=\frac{y U_{0}}{v}, U=\frac{u}{U_{0}}, V=\frac{v}{U_{0}}, \tau=\frac{t U_{0}^{2}}{v}, \theta=\frac{T-T_{\infty}}{T_{w}-T_{\infty}}, \phi=\frac{C-C_{\infty}}{C_{w}-C_{\infty}},
$$

So, $x=\frac{X v}{U_{0}}, y=\frac{Y v}{U_{0}}, u=U U_{0}, t=\frac{\tau v}{U_{0}^{2}}, T=T_{\infty}+\theta\left(T_{w}-T_{\infty}\right)$ and $C=C_{\infty}+\phi\left(C_{w}-C_{\infty}\right)$,

\section{Dimensionless Continuity Equation}

$$
U \frac{\partial U}{\partial X}+V \frac{\partial U}{\partial Y}=0
$$

\section{Dimensionless Momentum Equation}

$$
\frac{\partial U}{\partial \tau}+U \frac{\partial U}{\partial X}+V \frac{\partial U}{\partial Y}=G_{r} \theta+G_{c} \phi+\left(1+\frac{1}{\alpha}\right) \frac{\partial^{2} U}{\partial Y^{2}}-M U-\frac{1}{D_{a} U}
$$

\section{Dimensionless Energy Equation}

$$
\begin{aligned}
& \frac{\partial \theta}{\partial \tau}+\frac{\partial \theta}{\partial Y} U+V \frac{\partial \theta}{\partial Y}=\frac{1}{P_{r}}\left(1+\frac{16 R}{3}\right) \frac{\partial^{2} \theta}{\partial Y^{2}}+\left(1+\frac{1}{\alpha}\right) E_{c}\left(\frac{\partial U}{\partial Y}\right)^{2} \\
& +N_{b}\left(\frac{\partial \theta}{\partial Y} \frac{\partial C}{\partial Y}\right)+N_{t}\left(\frac{\partial \theta}{\partial Y}\right)^{2}
\end{aligned}
$$

\section{Dimensionless Concentration Equation}

$$
\begin{aligned}
& \frac{\partial \phi}{\partial \tau}+U \frac{\partial \phi}{\partial X}+V \frac{\partial \phi}{\partial Y}=\frac{1}{L_{e}}\left[\frac{\partial^{2} \phi}{\partial Y^{2}}+\left(\frac{N_{t}}{N_{b}}\right) \frac{\partial^{2} \theta}{\partial Y^{2}}\right] \\
& \tau \leq 0, U=0, V=0, \theta=0, \varphi=0 \text { every where } \\
& \tau>0, U=0, V=0, \theta=0, \varphi=0 \text { at } X=0 \\
& U=1, T=1, C=1 \quad \text { at } y=0 \\
& U=0, T=0, C=0 \quad \text { at } y \rightarrow \infty
\end{aligned}
$$

Grashof number, $G_{r}=\frac{g \beta\left(T_{w}-T_{\infty}\right) v}{U_{0}^{3}}$, mass Grashof number. $G_{r}=\frac{g \beta^{*}\left(C_{w}-C_{\infty}\right) v}{U_{0}^{3}}$, magnetic parameter, $M=\frac{\sigma^{\prime} B_{0}^{2} v}{\rho U_{0}^{2}}$, Darcy number, $D_{a}=\frac{\kappa U_{0}^{2}}{v^{2}}$, Prandtl number, $P_{r}=\frac{\rho c_{p} v}{\kappa}$, radiation parameter, $R=\frac{\sigma T_{\infty}^{3}}{k_{1} k}$, Eckert number, $\quad E_{c}=\frac{U_{0}^{2}}{c_{p}\left(T_{w}-T_{\infty}\right)} \quad$ Lewis number, $L e=\frac{v}{D_{m}}$, Brownian parameter, $N b=\frac{\tau D_{B}\left(C_{w}-C_{\infty}\right)}{v}$ and thermophoresis parameter, $N t=\frac{\tau D_{T}}{T_{\infty} v}\left(T_{w}-T_{\infty}\right)$.

Local shear stress $\tau_{L}=\mu\left(\frac{\partial u}{\partial y}\right)_{y=0}$ and average shear stress $\tau_{A}=\mu \int\left(\frac{\partial u}{\partial y}\right)_{y=0} d x$, which are proportional to $\left(\frac{\partial U}{\partial Y}\right)_{Y=0}$ and $\int_{0}^{100}\left(\frac{\partial U}{\partial Y}\right)_{Y=0} d X$ respectively. Local Nusselt number, $\quad N_{u L}=\mu\left(-\frac{\partial T}{\partial y}\right)_{y=0}$ and average Nusselt number, $N_{u A}=\mu \int\left(-\frac{\partial T}{\partial y}\right)_{y=0} d x$, which are proportional to $\left(-\frac{\partial \theta}{\partial Y}\right)_{Y=0}$ and $\int_{0}^{100}\left(-\frac{\partial \theta}{\partial Y}\right)_{Y=0} d X$ respectively. 
Local Sherwood number, $S_{h L}=\mu\left(-\frac{\partial C}{\partial y}\right)_{y=0}$ and Average Sherwood number, $S_{h A}=\mu \int\left(-\frac{\partial C}{\partial y}\right)_{y=0} d x$, which are proportional to $\left(-\frac{\partial \phi}{\partial Y}\right)_{Y=0}$ and $\int_{0}^{100}\left(-\frac{\partial \phi}{\partial Y}\right)_{Y=0} d X$ respectively.

\section{Numerical Solution}

The non-dimensional coupled partial differential equations have been solved by the associated initial and boundary conditions. The method of explicit finite difference has been used to solve (6) - (9) subject to the initial and boundary conditions. For this reason the area within the boundary layer is divided by some perpendicular lines of $Y$-axis, where the normal of the medium is $Y$ - axis as shown in Fig-2. It is assumed that the maximum length of boundary layer $Y_{\max }=20$ as corresponds to $Y \rightarrow \infty$. i.e. $Y$ vary from 0 to 20 and the number of grid spacing in $Y$ directions are $m(=100)$ and $n(=200)$, with the smaller time step $\Delta \tau=0.005$. Using the explicit finite difference approximation we have

Continuity Equation

$$
\frac{U_{i, j}-U_{i, j-1}}{\Delta X}+\frac{V_{i, j}-V_{i, j-1}}{\Delta Y}=0
$$

Momentum Equation

$$
\begin{aligned}
& \frac{U_{i, j}^{\prime}-U_{i, j}}{\Delta \tau}+U_{i, j} \frac{U_{i, j}-U_{i-1, j}}{\Delta X}+V_{i, j} \frac{U_{i, j+1}-U_{i-1, j}}{\Delta Y}=\frac{U_{i, j+1}-2 U_{i, j}+U_{i, j-1}}{(\Delta Y)^{2}} \\
& +\left(1+\frac{1}{\alpha}\right) \frac{U_{i, j+1}-2 U_{i, j}+U_{i, j-1}}{(\Delta Y)^{2}}-\left(M+\frac{1}{D_{a}}\right) U_{i, j}+G_{r} \theta_{i, j}+G_{c} \phi_{i, j}
\end{aligned}
$$

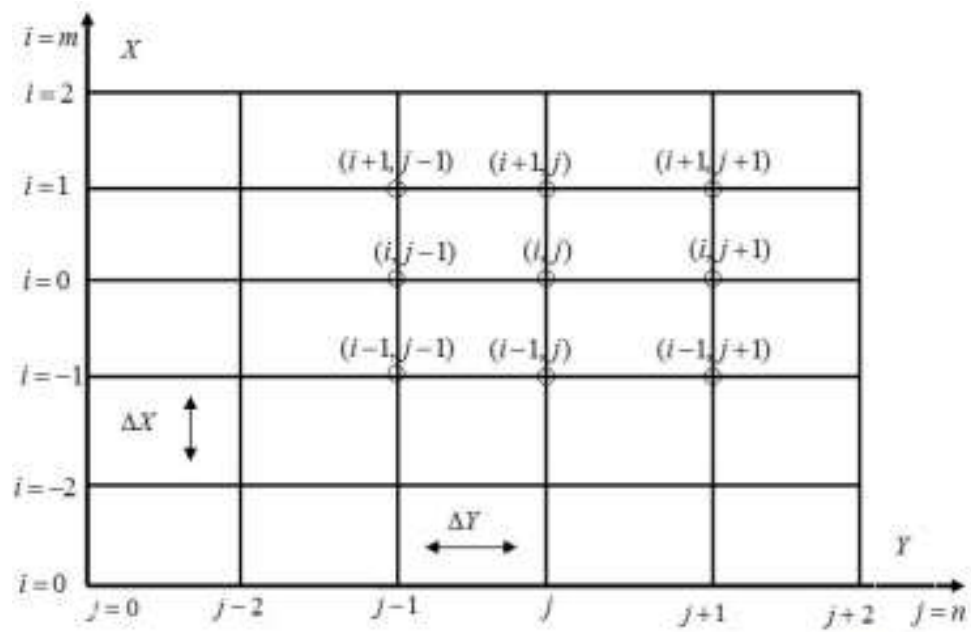

Fig-2: The finite difference space grid

Energy Equation

$$
\begin{aligned}
& \frac{\theta_{i, j}^{\prime}-\theta_{i, j}}{\Delta \tau}+U_{i, j} \frac{\theta_{i, j}-\theta_{i-1, j}}{\Delta X}+V_{i, j} \frac{\theta_{i, j+1}-\theta_{i-1, j}}{\Delta Y}=\frac{1}{P_{r}}\left(1+\frac{16}{3} R\right) \frac{\theta_{i, j+1}-2 \theta_{i, j}+\theta_{i, j-1}}{(\Delta Y)^{2}} \\
& +\left(1+\frac{1}{\alpha}\right) E_{c}\left(\frac{U_{i, j+1}-U_{i, j}}{\Delta Y}\right)^{2}+N b\left(\frac{\theta_{i, j+1}-\theta_{i, j}}{\Delta Y} \cdot \frac{\varphi_{i, j+1}-\varphi_{i, j}}{\Delta Y}\right)+N t\left(\frac{\theta_{i, j+1}-\theta_{i, j}}{\Delta Y}\right)^{2}
\end{aligned}
$$

Concentration Equation 


$$
\begin{aligned}
& \frac{\phi_{i, j}^{\prime}-\phi_{i, j}}{\Delta \tau}+U_{i, j} \frac{\phi_{i, j}-\phi_{i-1, j}}{\Delta X}+V_{i, j} \frac{\phi_{i, j+1}-\phi_{i-1, j}}{\Delta Y}=\frac{1}{L e}\left[\frac{\phi_{i, j+1}-2 \phi_{i, j}+\phi_{i, j-1}}{(\Delta Y)^{2}}\right. \\
& \left.+\left(\frac{N t}{N b}\right) \frac{\theta_{i, j+1}-2 \theta_{i, j}+\theta_{i, j-1}}{(\Delta Y)^{2}}\right]
\end{aligned}
$$

The initial and boundary condition with finite difference scheme as

$U_{i, 0}^{n}=1, \theta_{i, 0}^{n}=1, \phi_{i, 0}^{n}=1$

$U_{i, L}^{n}=0, \theta_{i, L}^{n}=0, \phi_{i, L}^{n}=0$ Where, $L \rightarrow \infty$

\section{Results and Discussion}

The fluid flow model solved by finite difference solution and obtained by explicit procedure. The numerical values of coupled non-dimensional velocity and temperature and concentration equation with the condition for different values of parameter have been computed by a FORTRAN program. Fig.1 and Fig.2 shows, velocity and temperature profiles decrease with the increase of Casson parameter $(\alpha)$. On the other hand in Fig.3, skin friction profiles increase for the increment of Casson parameter $(\alpha)$. From Fig.4 and Fig.5, we observe that velocity and temperature profiles increase near the plate and below $\mathrm{X}=0.5$ the curves intersect then decrease for the increment of Darcy number (Da). Fig. 6, Fig.7 and Fig.8 represent the velocity, temperature and concentration profiles for the increase of Lewis number (Le). Velocity and temperature profiles decrease and concentration profiles increase for increment of Lewis number (Le).

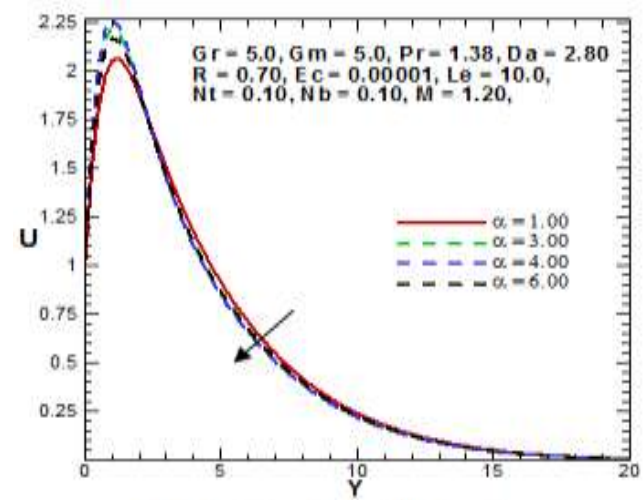

Fig. 1. Velocity profiles for $\alpha$

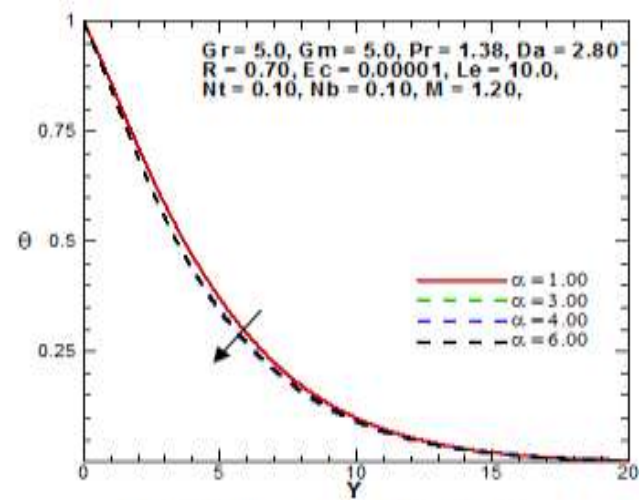

Fig. 2. Temperature profiles for $\alpha$

For the large impact of magnetic parameter (M) creates Lorentz for and for this reason the velocity profiles decrease in Fig.9. In Fig. 10 - Fig. 11 show that temperature profiles increase and concentration profiles decrease for the increase of Brownian parameter $(\mathrm{Nb})$. From Fig. 12- Fig. 13 show that temperature profiles decrease and concentration profiles increase for the increase of thermophoresis parameter $(\mathrm{Nt})$.

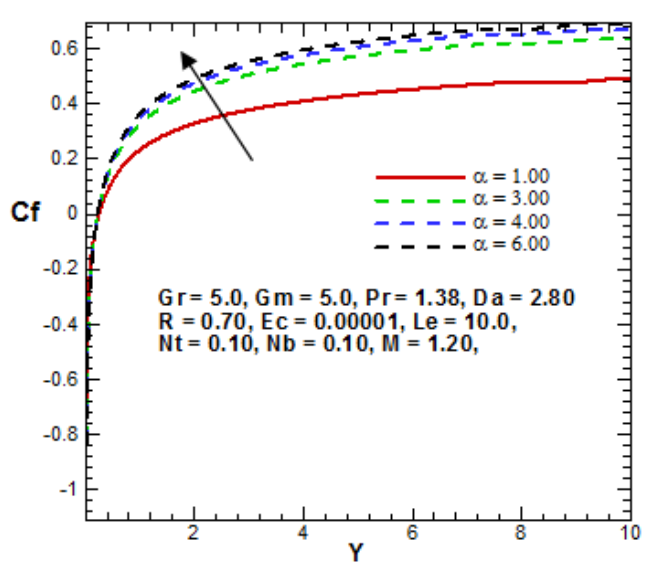

Fig. 3. Skin friction profiles for $\alpha$.

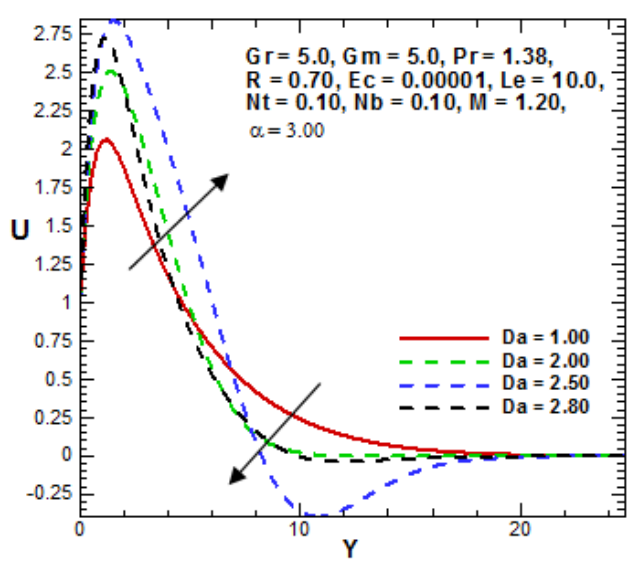

Fig. 4. Velocity profiles for $\mathrm{Da}$. 
Velocity and temperature profiles decrease for the increase of the Prandtl number (Pr) in Fig. 14Fig.15. Thermal radiation for a medium which contains it inevitably has pressure and density gradients and the treatment requires the use of hydrodynamics. For the electromagnetic radiation by the thermal excitation increase the fluid velocity for the effect of thermal radiation particles movement increase and for these reasons the temperature and skin friction profiles increase with the increase of thermal radiation (R) in Fig. 16 and Fig. 18. On the other hand in Fig. 17, Nusselt number $(\mathrm{Nu})$ profiles decrease for the increase of thermal radiation (R).

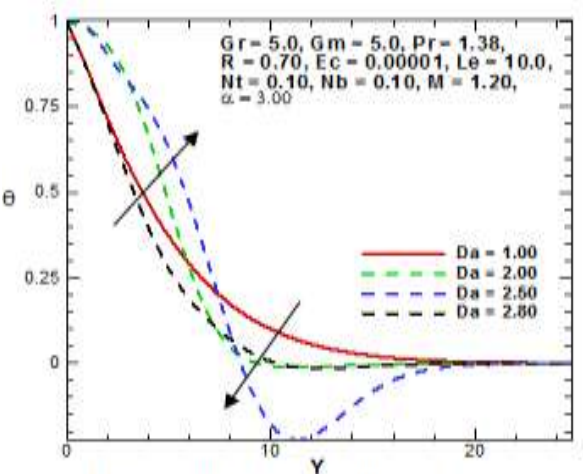

Fig. 5. Temperature profiles for $\mathrm{Da}$

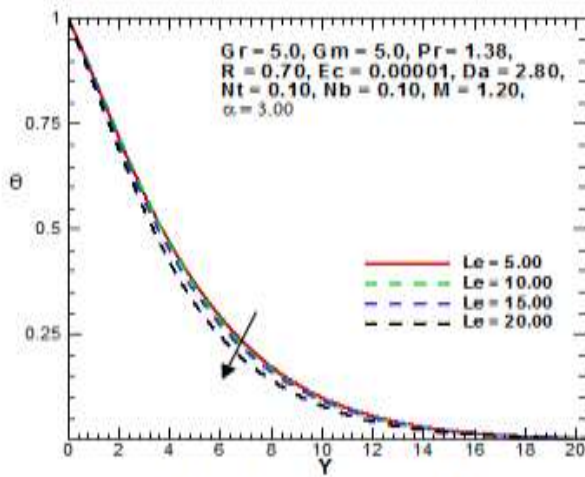

Fig. 7. Temperature profiles for Le

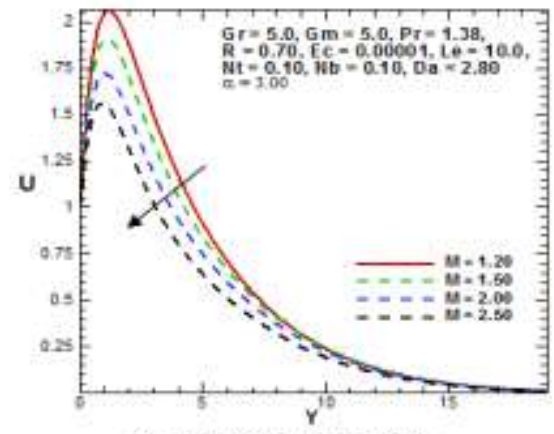

Fig. 9. Velocity profiles for $\mathrm{M}$

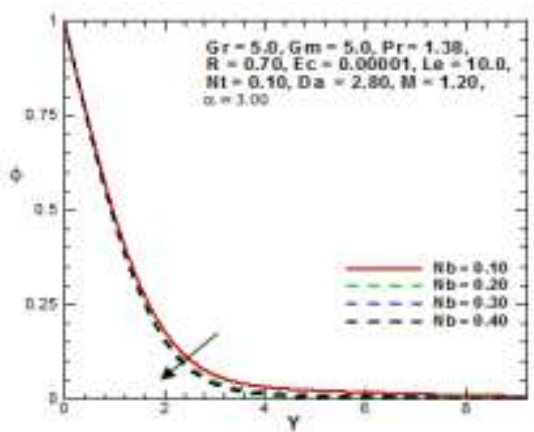

Fig. 11. Concentration profiles for $\mathrm{Nb}$

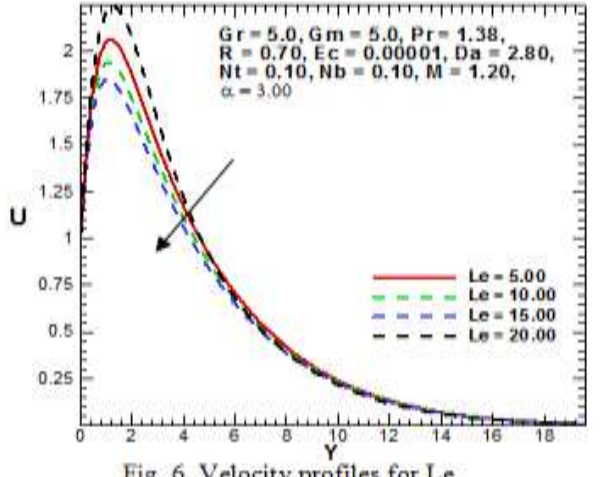

Fig. 6. Velocity profiles for Le.

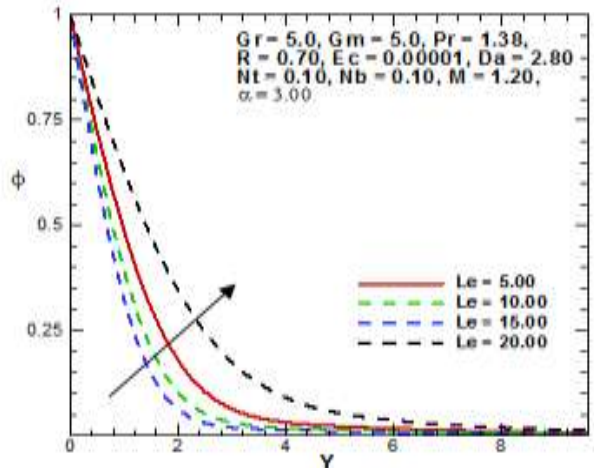

Fig. 8. Concentration profiles for Le.

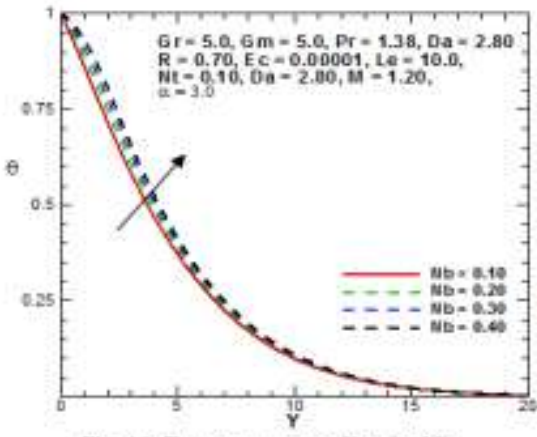

Fig. 10. Temperature profiles for $\mathrm{Nb}$

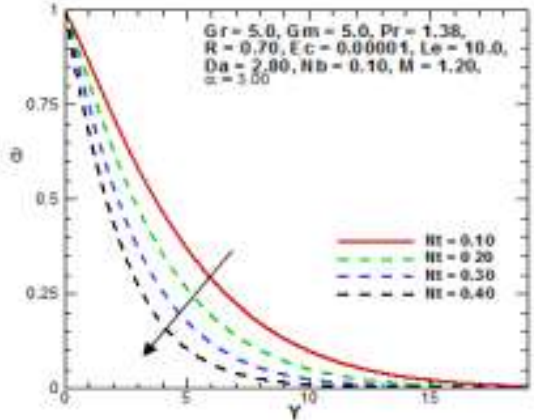

Fig 12. Temperature profiles for Ne. 


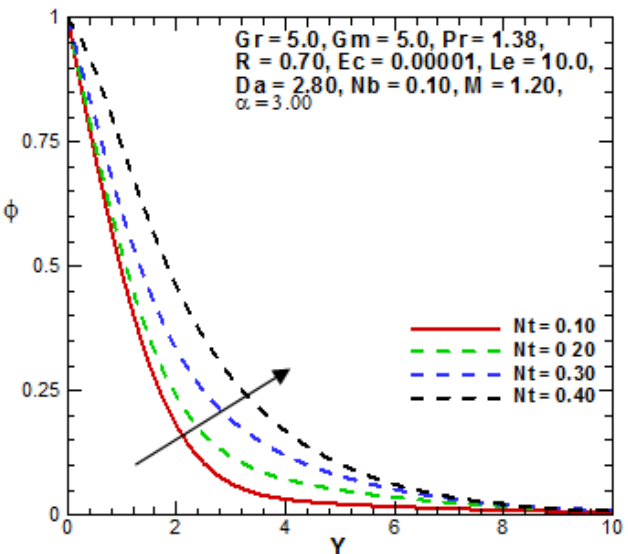

Fig. 13. Concentration profiles for $\mathrm{Nt}$.

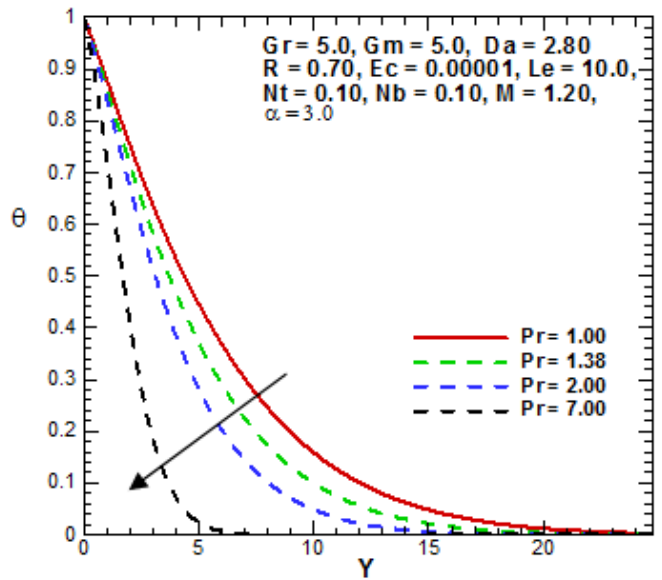

Fig. 15. Temperature profiles for Pr.

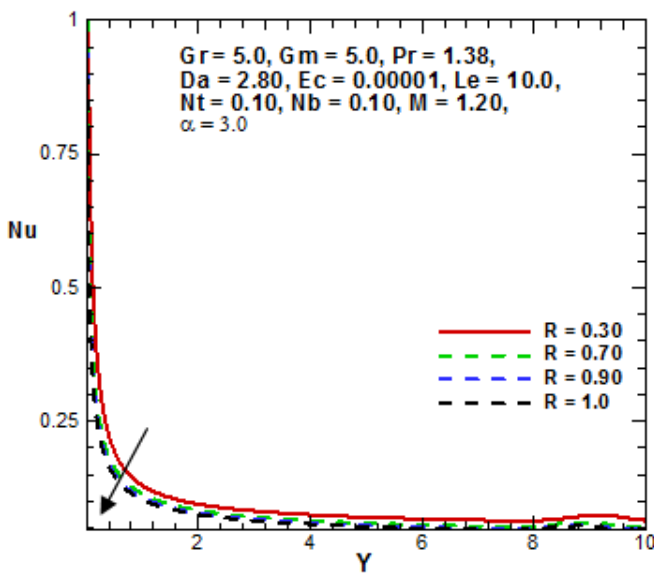

Fig. 17. Nusselt number profiles for $R$

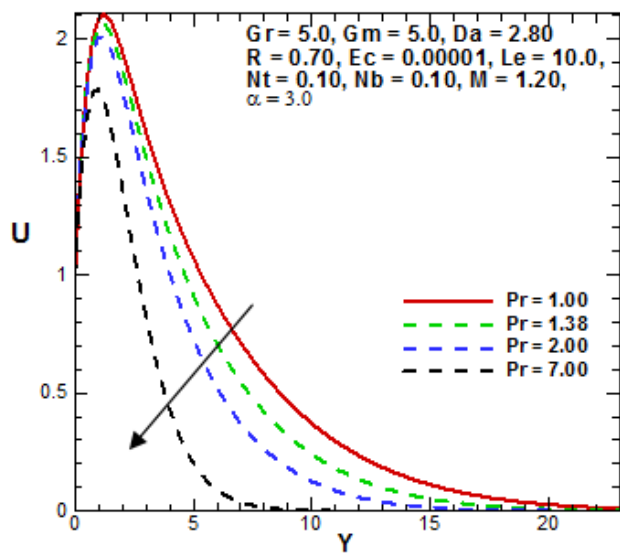

Fig. 14. Velocity profiles for $\operatorname{Pr}$

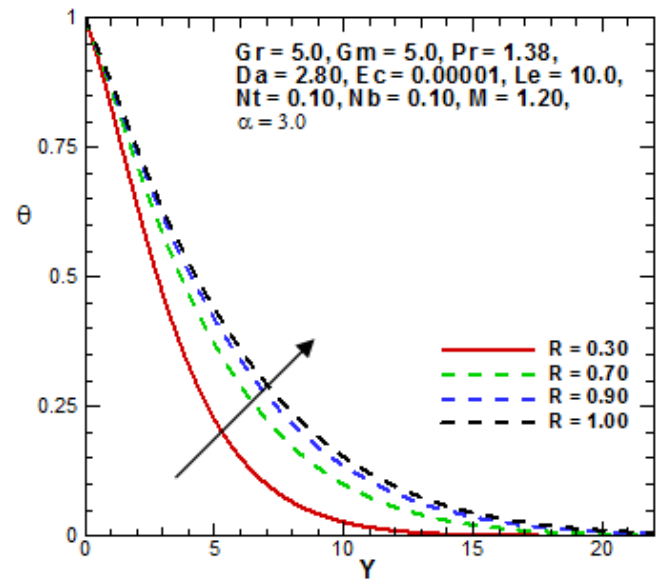

Fig. 16. Temperature profiles for $R$.

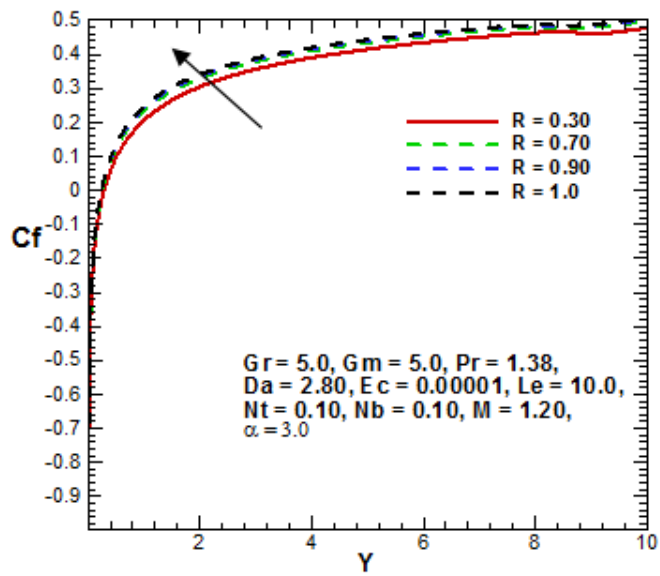

Fig. 18. Skin friction profiles for $\mathrm{R}$.

\section{Conclusion}

- Velocity and temperature profiles decrease with the increase of Casson parameter. But skin friction profiles increase.

- Near the plate, velocity and temperature profiles increase and faraway from the plate the profiles decrease for increment of Darcy number.

- Velocity and temperature profiles decrease and concentration profiles increase for of Lewis number.

- For the large values of magnetic parameter velocity profiles decrease.

- Temperature increase and concentration profiles decrease for the increment of Brownian parameter.

- Temperature decrease and concentration profiles increase for the increment of thermophoresis parameter. 
- Velocity and temperature profiles decrease for the increase of Prandtl number.

- Temperature and skin friction profiles increase but Nusselt number profiles decrease for the increase of thermal radiation.

\section{References}

[1]. Ibukun Sarah Oyelakin, Sabyasachi Mondal and Precious Sibanda (2016), Unsteady Casson nanofluid flow over a stretching sheet with thermal radiation, convective and slip boundary conditions, Alexandria Engineering Journal, 55, 1025-1035.

[2]. P.S. Reddy, A.J. Chamkha (2016), Soret and Dufour effects on MHD convective flow of Al2O3-water and TiO2-water nanofluids past a stretching sheet in porous media with heat generation/absorption, Advanced Powder Technology, 27, 1207-1218.

[3]. S. Nadeem, Rizwan Ul Haq and C. Lee (2012),MHD flow of a Casson fluid over an exponentially shrinking sheet, Scientia Iranica, 19:6, 1550-1553.

[4]. P.B.A. Reddy (2016), Magnetohydrodynamic flow of a Casson fluid over an exponentially inclined permeable stretching surface with thermal radiation and chemical reaction, Ain Shams Engineering Journal, 7, 593-602.

[5]. Swati Mukhopadhyay, Prativa Ranjan De, Krishnendu Bhattacharyya and G.C. Layek (2013), Casson fluid flow over an unsteady stretching surface, Ain Shams Engineering Journal, 4, 933-938.

[6]. A.H. Srinivasa, A.T. Eswara (2016), Effect of internal heat generation or absorption on MHD free convection from an isothermal truncated cone, Alexandria Engineering Journal, 55, 1367-1373.

[7]. T. Hayat, T. Muhammad, S.A. Shehzad, A. Alsaedi (2017), An analytical solution for magnetohydrodynamic Oldroyd-B nanofluid flow induced by a stretching sheet with heat generation/absorption, International Journal of Thermal Sciences, 111, 274-288.

[8]. M. E. Sayed-Ahmed, Hazem A. Attia and Karem M. Ewis (2011), Time Dependent Pressure Gradient Effect on Unsteady MHD Couette Flow and Heat Transfer of a Casson Fluid, Engineering, 3, 38-49.

[9]. S. Mosayebidorcheh, M. Sheikholeslami, M. Hatami, D.D. Ganji (2016), Analysis of turbulent MHD Couette nanofluid flow and heat transfer using hybrid DTM-FDM, Particuology, 26, 95-101.

[10]. Aminreza Noghrehabadi, Rashid Pourrajab and Mohammad Ghalambaz (2012), Effect of partial slip boundary condition on the flow and heat transfer of nanofluids past stretching sheet prescribed constant wall temperature, International Journal of Thermal Sciences, 54, 253-261.

[11]. S. Abdul Gaffar, V. Ramachandra Prasad, O. Anwar Be' g (2015), Numerical study of flow and heat transfer of non-Newtonian Tangent Hyperbolic fluid from a sphere with Biot number effects, Alexandria Engineering Journal 54, 829-841. 